\title{
The predictors of poor quality of life in a sample of Saudi women with breast cancer
}

This article was published in the following Dove Press journal:

Breast Cancer - Targets and Therapy

9 February 2017

Number of times this article has been viewed

\author{
Anwar E Ahmed ${ }^{1,2}$ \\ Abdulrahman G Alharbi ${ }^{3}$ \\ Mohannad A Alsadhan ${ }^{4}$ \\ Alaa S Almuzaini ${ }^{5}$ \\ Hanin S Almuzaini ${ }^{5}$ \\ Yosra Z Ali ${ }^{6}$ \\ Abdul-Rahman Jazieh ${ }^{6}$ \\ 'King Abdullah International Medical \\ Research Center (KAIMRC), \\ ${ }^{2}$ College of Public Health and \\ Health Informatics, King Saud bin \\ Abdulaziz University for Health \\ Sciences, ${ }^{3}$ College of Medicine, Imam \\ Abdulrahman Al Faisal University, \\ Dammam, ${ }^{4}$ College of Medicine, King \\ Saud University, ${ }^{5}$ College of Medicine, \\ King Saud bin Abdulaziz University \\ for Health Sciences, ${ }^{6}$ Department of \\ Oncology, King Abdulaziz Medical \\ City, Riyadh, Saudi Arabia
}

Background: The protocols for treatment, along with many adverse effects, can strongly affect cancer patients' quality of life (QoL). As there is limited research on the QoL of Saudi Arabian women being treated for breast cancer, the purpose of this study was to identify the predictors of poor QoL in a sample of Saudi women with breast cancer.

Methods: A cross-sectional study was conducted on 145 Saudi women with breast cancer who attended the Oncology Outpatient Clinic at King Abdulaziz Medical City, Riyadh for routine follow-up. Sociodemographic and clinical data were collected for each patient, and a Medical Outcome Study Health Survey 36-Item Short Form (SF-36) instrument was used to assess QoL. Results: Of 145 breast cancer patients studied, $42.1 \%$ had a family history of cancer and $52.4 \%$ were newly diagnosed cancer patients (first-year-after-cancer diagnosis). According to linear regression analyses, cancer patients with metastasis tended to have pain, along with poor physical function, little vitality, and poor general health (a decrease in SF-36 scores of 22.9, 15.0, 19.4, and 16.9, respectively). Regular exercise was a positive predictor of poor general health (an increase in the SF-36 score of 8.2). Patients with first-year-after-cancer diagnoses tended to have poor emotional well-being (a decrease in the SF-36 score of 8.5).

Conclusion: In breast cancer patients, regular exercise was a significant positive predictor of better general health. Breast cancer patients with multiple tumors, metastasis, or fever tended to experience significantly poor QoL in several SF-36 domains. Clearly, a routine assessment of QoL in breast cancer patients is important.

Keywords: multiple tumors, fever, metastasis, regular exercise, SF-36

\section{Introduction}

In most countries of the world, breast cancer is the most prevalent cancer. ${ }^{1,2}$ Saudi Arabia is no exception: breast cancer is reported to be the most common cancer in Saudi women, particularly among women aged $\geq 45$ years. ${ }^{3}$ The average Saudi woman's age at diagnosis is 49 years. ${ }^{3}$ New cases of breast cancer among Saudi women have increased from 1,430 in $1996^{3,4}$ to 1,542 in $2012 .{ }^{4}$ Later, the kingdom's Ministry of Health reported that 2,741 new breast cancer cases were diagnosed in Saudi Arabia. The Saudi National Cancer Registry reported an increase of breast cancer among Saudi women from $19.1 \%$ (based on a 1996 report) to $25.8 \%$ (based on a 2012 report), including all newly diagnosed cancer tumors. The incidence rates of breast cancer vary by geographic regions: 32.6 per 100,000 in Eastern, 31.6 per 100,000 in Jouf, and 25.6 per 100,000 in Riyadh. ${ }^{4}$

There are several treatment options used worldwide for breast cancer. The choices may depend on the type of breast cancer, stage, tumor size, and the patient's
Correspondence: Anwar E Ahmed College of Public Health and Health Informatics, King Saud bin Abdulaziz University for Health Sciences, MC 2350, P.O. Box 22490, Riyadh II426, KSA, Saudi Arabia

Email ahmeda5@vcu.edu 
demographics and reactions to hormones. In Saudi Arabia, treatment of breast cancer may involve surgery, antibodies, chemotherapy, hormone therapy, and radiation, or there could be a combination of treatments. Patients with breast cancer tend to experience a poor quality of life (QoL) during or after the treatment course. ${ }^{5}$ As the result of a mastectomy, women with breast cancer reported a poor self-image 1 month after surgery and a poor QoL 1 year after the cancer diagnosis. ${ }^{6}$ A study of 86 patients reported that breast cancer may affect a woman's sexual activity. ${ }^{7}$ A study in Australia assessed the impact of breast cancer and surgery on QoL classified by age and marital status. According to the authors, women who were young, single, and lacked education reported worse QoL than older, married, and educated women. ${ }^{8}$ Furthermore, a mastectomy tended to have a negative effect on body image among married women. ${ }^{8,9}$ Another study reported that $>70 \%$ of young women experienced pain and unhappiness with regard to appearance. ${ }^{10}$ Breast cancer patients commonly experience major psychological symptoms, including depression, anxiety, and stress. ${ }^{1-14}$

A 2011 study reveals that Arab women with breast cancer, especially those receiving little support and those with a poor body image, experienced powerful psychological distress. ${ }^{15} \mathrm{~A}$ different study reported that young Arab women experienced more negative emotions regarding their illnesses than older Arab women. ${ }^{16}$ There are several studies that assessed QoL in Arab breast cancer patients in various countries: Egypt, ${ }^{17-19}$ United Arab Emirates, ${ }^{20}$ Kuwait, ${ }^{21}$ Tunisia, ${ }^{22}$ Yemen, ${ }^{23}$ Lebanon, ${ }^{24}$ Bahrain, ${ }^{25,26}$ Morocco, ${ }^{27}$ and Jordan. ${ }^{28}$ There is little data on the connection between breast cancer and QoL among Saudi women, where only a single study has assessed the QoL among breast cancer patients in Saudi Arabia. ${ }^{29}$ This study in Riyadh, Saudi Arabia, used the European Organisation for Research and Treatment of Cancer to measure QoL of breast cancer patients who were recruited from different outpatient clinical settings. There appears to be poor overall global QoL ${ }^{29}$ but this study did not assess the influence of the side effects of treatment and other clinical factors on QoL. Today, however, placing attention on cancer patients is increasingly common worldwide, as it allows researchers to introduce intervention to reduce the psychological effects of the diagnosis or treatment.

Despite this earlier lack of attention, there are several studies in the literature assessing attitude, awareness, and knowledge of breast cancer and its self-examination among Saudi women in various regions. ${ }^{30-37}$ However, not much is known about the influence of the side effects of treatment and other clinical factors on the QoL of Saudi women with breast cancer. Clinicians would benefit from this study by promptly being able to assess the QoL in order to provide proper treatment.

\section{Purpose}

The purpose of this study is to efficiently assess the QoL of Saudi women with breast cancer when compared with sociodemographic factors. We hypothesized that breast cancer in Saudi women may negatively affect their QoL, and we also investigated whether QoL depends on any sociodemographic factors.

\section{Methods}

A cross-sectional study was conducted in the Oncology Outpatient Clinic at King Abdulaziz Medical City, Riyadh, Ministry of National Guard Health Affairs, where we assessed QoL in a sample of female breast cancer patients. The study received ethical approval from the Institutional Review Board (IRB) at King Abdullah International Medical Research Center, IRB approval \# RSS16/004. A consecutive sample of women with breast cancer who were seen in the Oncology Outpatient Clinic during the study period (August 14-31, 2016) were asked to participate in a one-time survey to assess QoL and psychological symptoms. The participants of the study received a survey with a cover letter explaining the aims of the study and asking whether they wanted to participate. Only patients who agreed to participate in the study were included and asked to complete a survey. We excluded breast cancer patients who had been referred to palliative care. We distributed 170 questionnaires to women with breast cancer who attended the oncology clinic during the study period, and 145 (85.3\%) questionnaires were completed and returned. Completion of survey is deemed to be agreement of consent from the 145 participants.

The first section of the survey covers sociodemographic characteristics. We asked women about their age, weight, height, marital status, education level, employment status, monthly income category, physical exercise, and whether they received family support. Furthermore, we collected data about patient clinical and cancer characteristics (eg, comorbidity factors, types of treatment received to treat breast cancer, cancer grade, metastasis, fever, and chronic disease other than cancer).

\section{Study instruments}

In this study, we assessed the QoL of breast cancer patients using the Medical Outcome Study Health Survey 36-Item Short Form (SF-36). ${ }^{38}$ The questionnaire was found to be reliable and valid and its Arabic version is publicly available. ${ }^{39}$ The SF-36 is comprised of items addressing eight health 
concepts: role limitation due to physical health, physical functioning, role limitations due to emotional problems, social functioning, vitality, bodily pain, emotional well-being, and general health perceptions. SF-36 items were scored based on the RAND Health Scoring System. ${ }^{38}$ For example, the total of each mentioned domain ranges from $0=$ poor to $100=$ best health. In our population, the SF-36 subscales were reliable and demonstrated good internal consistency: physical functioning (Cronbach's alpha=0.91), role limitations due to physical health (Cronbach's alpha $=0.86$ ), role limitations due to emotional problems (Cronbach's alpha $=0.87$ ), vitality (Cronbach's alpha=0.74), emotional well-being (Cronbach's alpha $=0.80$ ), social functioning (Cronbach's alpha=0.87), pain (Cronbach's alpha $=0.88$ ), and general health (Cronbach's alpha=0.69).

\section{Statistical analyses}

The data analyses were performed using IBM SPSS Statistics 23 (IBM Corp, Armonk, NY, USA).

\section{Patients' sociodemographics}

Descriptive statistics, such as mean and standard deviation (mean $\pm \mathrm{SD}$ ), were used to describe the quantitative variables. Frequencies and percentages n (\%) were used to describe categorical variables (Table 1).

\section{Bivariate analyses}

To account for 19 multiple comparisons, the Bonferroni correction of $\alpha / n=0.05 / 19=0.003$ was used to compare QoL between the groups (Tables 1 and 2). Specifically, we will compare physical functioning, role limitations due to physical health, role limitations due to emotional problems, vitality, social functioning, bodily pain, general health, physical wellbeing, social/family well-being, emotional well-being, and functional well-being perceptions across sociodemographic and clinical characteristics.

\section{Multivariate analyses}

Multiple linear regression models were used to identify predictors of the SF-36 subscales (Table 3). The regression coefficients will be used to interpret the linear regression findings. In all multivariate analyses, the significance level (a) was set at 0.05 .

\section{Results}

Our analysis included 145 female patients with breast cancer. The sample studied was relatively old. The mean age was $50.3( \pm \mathrm{SD}=13.5)$ years with an age range between 21 and 87 years, and $22.1 \%$ were elderly women aged $\geq 60$ years. Of the sample studied, $42.1 \%$ had a family history of cancer, $74.5 \%$ were unemployed, $37.2 \%$ were obese, and $25.5 \%$ reported having multiple tumors. Other clinical and demographic details are shown in Table 1 . The median number of months after breast cancer diagnosis was 12 with percentiles $(q 1=6$, q3=24). Differences across sociodemographic and clinical data are shown for all eight domains in Tables 1 and 2. Breast cancer patients who were elderly, with high school education or less, had multiple tumors, had metastasis, received immunotherapy reported poor physical functions. Breast cancer patients having multiple tumors, metastasis, and fever had lower scores regarding role limitations due to emotional problems. Breast cancer patients having multiple tumors and metastasis reported poor vitality. Breast cancer patients with fever reported low scores regarding emotional well-being. Social functioning was poorer in breast cancer patients with multiple tumors and metastasis (Table 2). We noted worse bodily pain in breast cancer patients with late-stage cancer (III or IV), multiple tumors, metastasis, and fever (Figure 1). We discovered poor general health in patients with multiple tumors, metastasis, and fever. However, the general health of patients was better in patients who regularly exercised.

According to linear regression analyses (Table 3), breast cancer patients with a fever tended to have worse emotional well-being and social function (a decrease in SF-36 scores of 9.1 and 11.0, respectively) when compared with breast cancer patients with no fever. Metastasis was predictive of decreasing physical function, vitality, general health, and increasing bodily pain (with scores of 22.9, 15.0, 16.9, and 19.4, respectively). Employment was predictive of worsening role limitations due to emotional problems, vitality, and emotional well-being (a decrease in SF-36 scores of 20.4, 9.4, and 10.1, respectively). Breast cancer patients who received immunotherapy were predicted to have less vitality and increased pain by scores of 7.2 and 10.8, respectively. Multiple tumors were predicted to worsen role limitations due to emotional problems and social function by scores of 31.6 and 20.6, respectively. Newly diagnosed breast cancer patients with first-year-after-diagnosis tended to have a decrease in social function by a score of 14.2. However, regular exercise was predictive of better general health by an increased score of 8.2. Social function tended to decrease by 0.4 as age increased by one year.

\section{Discussion}

The aim of this study was to assess the QoL of Saudi women with breast cancer and compare it with their sociodemographic 
Table I Differences in quality of life by demographic and clinical characteristics $(N=\mid 45)$

\begin{tabular}{|c|c|c|c|c|c|c|c|c|}
\hline \multirow[t]{2}{*}{ Characteristics } & \multirow[t]{2}{*}{ Levels } & \multirow{2}{*}{$\begin{array}{l}\text { Overall, } \\
\mathrm{N}=145 \\
\mathrm{n}(\%)\end{array}$} & \multicolumn{2}{|c|}{$\begin{array}{l}\text { Physical functioning, } \\
54.5 \pm 28.8\end{array}$} & \multicolumn{2}{|c|}{$\begin{array}{l}\text { Role limitations due } \\
\text { to physical health, } \\
29.3 \pm 38.3\end{array}$} & \multicolumn{2}{|c|}{$\begin{array}{l}\text { Role limitations due } \\
\text { to emotional problems, } \\
33.6 \pm 42 \text {.I }\end{array}$} \\
\hline & & & Mean (SD) & $P$-value & Mean (SD) & $P$-value & Mean (SD) & $P$-value \\
\hline \multirow[t]{2}{*}{ Elderly } & No & II 3 (77.9) & $58.4(27.6)$ & $0.002 *$ & $27.4(37.6)$ & 0.270 & $3 I .9(4 \mid .2)$ & 0.363 \\
\hline & Yes & $32(22.1)$ & $40.5(28.7)$ & & $35.9(40.6)$ & & $39.6(45.9)$ & \\
\hline \multirow[t]{2}{*}{ University } & No & $98(67.6)$ & $48.7(26.8)$ & $0.001 *$ & 30.1 (38.0) & 0.721 & 34.0 (4I.7) & 0.854 \\
\hline & Yes & $47(32.4)$ & $66.4(29.2)$ & & 27.7 (39.4) & & $32.6(43.7)$ & \\
\hline \multirow[t]{2}{*}{ Employed } & No & $108(74.5)$ & 5I.I (27.0) & 0.015 & $31.0(38.6)$ & 0.361 & $37.3(43.6)$ & 0.045 \\
\hline & Yes & $37(25.5)$ & $64.3(31.6)$ & & $24.3(37.5)$ & & $22.5(36.1)$ & \\
\hline \multirow[t]{2}{*}{ Married } & No & $37(25.5)$ & 49.1 (28.9) & 0.187 & 3I.I (36.5) & 0.746 & $31.5(38.4)$ & 0.736 \\
\hline & Yes & $108(74.5)$ & $56.3(28.6)$ & & $28.7(39.1)$ & & $34.3(43.6)$ & \\
\hline \multirow[t]{2}{*}{ Obese } & No & $76(62.8)$ & $53.9(28.3)$ & 0.741 & $34.2(4 I .4)$ & 0.194 & $34.2(44.2)$ & 0.869 \\
\hline & Yes & $45(37.2)$ & $55.8(3 \mid .2)$ & & $25.0(35.0)$ & & $35.6(41.7)$ & \\
\hline \multirow{2}{*}{$\begin{array}{l}\text { Family history of } \\
\text { cancer }\end{array}$} & No & 84 (57.9) & $54.0(29.1)$ & 0.882 & $35.1(40.2)$ & 0.028 & $38.5(44.7)$ & 0.091 \\
\hline & Yes & $6 I(42.1)$ & $55.1(28.4)$ & & $21.3(34.4)$ & & $26.8(37.9)$ & \\
\hline \multirow{2}{*}{$\begin{array}{l}\text { First-year-after-cancer } \\
\text { diagnosis }\end{array}$} & No & $69(47.6)$ & $52.4(27.3)$ & 0.414 & $25.7(35.1)$ & $0.28 \mathrm{I}$ & $32.4(40.4)$ & 0.745 \\
\hline & Yes & $76(52.4)$ & $56.3(30.0)$ & & $32.6(4 I .0)$ & & $34.6(44.0)$ & \\
\hline \multirow[t]{2}{*}{ Cancer stage } & $1 / I I$ & $79(6 \mid .2)$ & $61.5(27.6)$ & 0.005 & $31.3(38.3)$ & 0.489 & $40.1(44.1)$ & 0.016 \\
\hline & III/IV & $50(38.8)$ & $47.0(28.2)$ & & $26.5(38.9)$ & & 22.7 (35.9) & \\
\hline \multirow[t]{3}{*}{ Multiple tumors } & No & $108(74.5)$ & $58.9(27.9)$ & $0.001 *$ & $32.2(39.7)$ & 0.095 & $4 I .4(44.4)$ & $0.001 *$ \\
\hline & Yes & $37(25.5)$ & $41.5(27.5)$ & & $20.9(33.1)$ & & 10.8 & \\
\hline & & & & & & & $(23.6)$ & \\
\hline \multirow[t]{2}{*}{ Cancer surgery } & No & $51(35.2)$ & $56.2(30.9)$ & 0.596 & $33.8(40.9)$ & 0.298 & $3 I .4(4 \mid .3)$ & 0.647 \\
\hline & Yes & $94(64.8)$ & $53.5(27.6)$ & & $26.9(36.9)$ & & $34.8(42.9)$ & \\
\hline \multirow[t]{2}{*}{ Chemotherapy } & No & $39(26.9)$ & $55.0(28.4)$ & 0.889 & $34.6(39.2)$ & 0.314 & $35.9(44.2)$ & 0.688 \\
\hline & Yes & $106(73.1)$ & $54.2(29.0)$ & & $27.4(38.0)$ & & $32.7(4 \mid .7)$ & \\
\hline \multirow[t]{2}{*}{ Radiation therapy } & No & $6 \mathrm{I}(42.1)$ & $52.8(30.6)$ & 0.555 & $30.7(38.8)$ & 0.704 & $27.3(37.8)$ & 0.120 \\
\hline & Yes & $84(57.9)$ & $55.7(27.5)$ & & $28.3(38.2)$ & & $38.1(44.9)$ & \\
\hline \multirow[t]{2}{*}{ Immunotherapy } & No & $74(5 \mid .0)$ & $61.8(28.0)$ & $0.001 *$ & $33.8(4 I .7)$ & 0.151 & $39.6(44.7)$ & 0.076 \\
\hline & Yes & $71(49.0)$ & $46.8(27.7)$ & & $24.6(34.2)$ & & $27.2(38.8)$ & \\
\hline \multirow[t]{2}{*}{ Antibodies } & No & $102(72.3)$ & $57.3(27.5)$ & 0.074 & $29.7(38.0)$ & 0.949 & $35.9(43.4)$ & 0.453 \\
\hline & Yes & $39(27.7)$ & $47.6(31.6)$ & & $30.1(40.6)$ & & $29.9(40.3)$ & \\
\hline \multirow[t]{2}{*}{ Metastasis } & No & $101(69.7)$ & $60.3(26.4)$ & $0.001 *$ & $31.4(38.8)$ & 0.314 & $41.6(44.6)$ & $0.001 *$ \\
\hline & Yes & $44(30.3)$ & $40.9(29.7)$ & & $24.4(37.2)$ & & $15.2(29.2)$ & \\
\hline \multirow[t]{2}{*}{ Fever } & No & $91(62.8)$ & $56.1(30.1)$ & 0.371 & $33.5(39.8)$ & 0.086 & $41.0(44.7)$ & $0.003^{*}$ \\
\hline & Yes & $54(37.2)$ & $51.7(26.3)$ & & $22.2(34.9)$ & & $21.0(34.4)$ & \\
\hline \multirow[t]{2}{*}{ Family support } & No & $14(9.7)$ & $62.9(32.0)$ & 0.251 & $21.4(33.8)$ & 0.420 & $31.0(46.2)$ & 0.809 \\
\hline & Yes & $13 \mid(90.3)$ & $53.5(28.4)$ & & $30.2(38.8)$ & & $33.8(42.0)$ & \\
\hline \multirow{2}{*}{$\begin{array}{l}\text { Chronic disease other } \\
\text { than cancer }\end{array}$} & No & $93(64.1)$ & $59.3(27.9)$ & 0.006 & $30.1(40.0)$ & 0.739 & $31.2(4 I .6)$ & 0.366 \\
\hline & Yes & $52(35.9)$ & $45.8(28.5)$ & & $27.9(35.6)$ & & $37.8(43.3)$ & \\
\hline Regular exercise & No & $99(68.3)$ & $51.8(29.5)$ & 0.106 & $29.0(39.7)$ & 0.902 & $32.3(43.0)$ & 0.606 \\
\hline & Yes & $46(31.7)$ & $60.1(26.6)$ & & $29.9(35.6)$ & & $36.2(40.9)$ & \\
\hline
\end{tabular}

Note: $*$ The variable is significant using the Bonferroni correction cutoff at $\alpha / n=0.05 / 19=0.003$, where $n$ is the number of tests.

Abbreviation: SD, standard deviation.

and clinical factors. Despite the fact that QoL assessment has now become part of cancer clinical research trials and studies, there is very limited data existing on QoL in Saudi Arabia. We noted low SF-36 scores with minimum average values of 29.3 (role limitations due to physical health) and a maximum average value of 61.4 (emotional well-being). However, these scores were lower than those found in Saudi women with sickle cell disease, except for pain and emotional well-being where both populations reported similar scores ${ }^{40}$ The study supports evidence of findings in the existing literature, which indicates that breast cancer survivors report poor QoL. A study in Saudi Arabia showed breast cancer survivors reported a low overall global QoL, ${ }^{29}$ whereas Chinese and U.S. breast cancer survivors reported poor QoL. ${ }^{41}$ A study in Sweden reported poorer QoL in newly diagnosed breast cancer patients when compared with the normative population..$^{42}$ Our study suggested that employment was a negative predictor of role limitations due to emotional problems, vitality, and emotional well-being.

In this study, significant differences were observed in physical functions when measured by age groups and 
Table 2 Differences in quality of life by demographic and clinical characteristics $(N=\mid 45)$

\begin{tabular}{|c|c|c|c|c|c|c|c|c|c|c|c|}
\hline \multicolumn{2}{|l|}{ Characteristics } & \multirow{2}{*}{$\begin{array}{l}\text { Vitality, } \\
43.9 \pm 20.8 \\
\text { Mean (SD) }\end{array}$} & \multirow[t]{2}{*}{$P$-value } & \multirow{2}{*}{$\begin{array}{l}\text { Emotional } \\
\text { wellbeing, } \\
61.4 \pm 20.0 \\
\text { Mean (SD) }\end{array}$} & \multirow[t]{2}{*}{$P$-value } & \multirow{2}{*}{$\begin{array}{l}\text { Social } \\
\text { functioning, } \\
58.4 \pm 28.2 \\
\text { Mean (SD) }\end{array}$} & \multirow[t]{2}{*}{$P$-value } & \multirow{2}{*}{$\begin{array}{l}\text { Pain, } \\
49.0 \pm 26.6 \\
\text { Mean (SD) }\end{array}$} & \multirow[t]{2}{*}{$P$-value } & \multirow{2}{*}{ 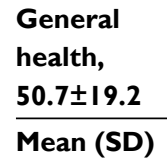 } & \multirow[t]{2}{*}{$P$-value } \\
\hline & & & & & & & & & & & \\
\hline \multirow[t]{2}{*}{ Elderly } & No & $44.2(20.3)$ & 0.740 & $59.8(20.6)$ & 0.062 & $59.0(28.1)$ & 0.633 & $48.5(27.1)$ & 0.632 & $51.3(19.2)$ & 0.486 \\
\hline & Yes & $42.8(22.7)$ & & $67.3(17.0)$ & & $56.3(28.9)$ & & $51.0(25.2)$ & & $48.6(19.3)$ & \\
\hline \multirow[t]{2}{*}{ University } & No & $43.4(21.4)$ & 0.660 & $63.0(19.6)$ & 0.162 & 6I.I (28.8) & 0.092 & $47.6(27.1)$ & 0.349 & $50.9(18.9)$ & 0.837 \\
\hline & Yes & $45.0(19.6)$ & & $58.0(20.7)$ & & $52.7(26.4)$ & & $52.0(25.5)$ & & $50.2(19.9)$ & \\
\hline \multirow[t]{2}{*}{ Employed } & No & $44.9(21.0)$ & 0.319 & 63.7 (19.0) & 0.020 & 6I.I (26.7) & 0.045 & $49.6(25.8)$ & 0.651 & $50.7(18.5)$ & 0.996 \\
\hline & Yes & $40.9(20.3)$ & & $54.8(21.7)$ & & $50.3(31.4)$ & & $47.3(29.2)$ & & $50.7(21.4)$ & \\
\hline \multirow[t]{2}{*}{ Married } & No & 44.5 (19.2) & 0.850 & $63.5(13.6)$ & 0.371 & $59.8(26.0)$ & 0.721 & $50.1(24.6)$ & 0.768 & $52.8(18.9)$ & 0.432 \\
\hline & Yes & $43.7(21.4)$ & & $60.7(21.8)$ & & $57.9(29.0)$ & & $48.6(27.4)$ & & $50.0(19.3)$ & \\
\hline \multirow[t]{2}{*}{ Obese } & No & 44.5 (19.8) & 0.631 & $61.3(18.9)$ & 0.918 & $57.2(28.7)$ & 0.495 & $49.7(28.7)$ & 0.749 & $50.5(17.8)$ & 0.982 \\
\hline & Yes & $42.7(22.1)$ & & $60.9(20.0)$ & & $60.8(26.5)$ & & $48.1(23.1)$ & & $50.4(20.7)$ & \\
\hline \multirow{2}{*}{$\begin{array}{l}\text { Family history of } \\
\text { cancer }\end{array}$} & No & $46.0(21.3)$ & 0.151 & $64.0(19.9)$ & 0.073 & $63.1(28.1)$ & 0.017 & $52.8(28.1)$ & 0.039 & $51.6(19.0)$ & 0.501 \\
\hline & Yes & 41.0 (19.9) & & $57.9(19.9)$ & & $51.8(27.3)$ & & $43.8(23.7)$ & & 49.4 (19.5) & \\
\hline First-year-after- & No & 43.4 (23.7) & 0.791 & $63.7(21.3)$ & 0.200 & 62.7 (29.9) & 0.079 & $49.7(29.4)$ & 0.766 & $52.9(18.3)$ & 0.188 \\
\hline cancer diagnosis & Yes & $44.3(17.9)$ & & $59.4(18.7)$ & & $54.4(26.2)$ & & $48.4(24.0)$ & & 48.7 (19.9) & \\
\hline \multirow[t]{2}{*}{ Cancer stage } & $\mathrm{I} / \mathrm{II}$ & 47.7 (I8.8) & 0.005 & $63.0(19.7)$ & 0.086 & $62.2(24.1)$ & 0.114 & $54.2(24.7)$ & $0.002^{*}$ & $53.7(18.4)$ & 0.008 \\
\hline & $\mathrm{III} / \mathrm{IV}$ & $37.6(21.3)$ & & $56.8(19.8)$ & & $53.8(32.1)$ & & $39.9(26.6)$ & & 44.6 (I8.7) & \\
\hline \multirow[t]{2}{*}{ Multiple tumors } & No & $47.2(20.3)$ & $0.00 I^{*}$ & $63.3(20.1)$ & 0.052 & $62.7(26.7)$ & $0.00 I^{*}$ & $54.3(26.1)$ & $0.00 I^{*}$ & $54.1(18.3)$ & $0.001 *$ \\
\hline & Yes & $34.2(19.3)$ & & $55.9(19.0)$ & & $45.6(28.9)$ & & $33.6(22.1)$ & & $40.8(18.6)$ & \\
\hline \multirow[t]{2}{*}{ Cancer surgery } & No & $43.0(17.3)$ & 0.716 & $63.2(14.5)$ & 0.367 & $55.1(26.8)$ & 0.314 & 49.5 (23.7) & 0.870 & $46.6(18.1)$ & 0.057 \\
\hline & Yes & $44.4(22.6)$ & & $60.4(22.5)$ & & $60.1(28.9)$ & & $48.8(28.2)$ & & $52.9(19.5)$ & \\
\hline \multirow[t]{2}{*}{ Chemotherapy } & No & $45.0(18.8)$ & 0.700 & $62.1(21.9)$ & 0.815 & $59.3(25.9)$ & 0.810 & $51.5(27.4)$ & 0.502 & $50.1(22.2)$ & 0.832 \\
\hline & Yes & $43.5(21.6)$ & & $61.2(19.4)$ & & $58.0(29.1)$ & & $48.1(26.4)$ & & $50.9(18.1)$ & \\
\hline \multirow[t]{2}{*}{ Radiation therapy } & No & $43.3(19.2)$ & 0.762 & $62.8(18.0)$ & 0.472 & $56.8(27.3)$ & 0.563 & 49.7 (22.9) & 0.782 & 50.7 (19.9) & 0.980 \\
\hline & Yes & $44.3(22.0)$ & & $60.4(21.5)$ & & $59.5(29.0)$ & & $48.5(29.2)$ & & $50.7(18.8)$ & \\
\hline \multirow[t]{2}{*}{ Immunotherapy } & No & $48.5(16.6)$ & 0.006 & $63.0(18.0)$ & 0.339 & $57.9(23.8)$ & 0.856 & $55.2(23.5)$ & 0.004 & $52.0(19.0)$ & 0.418 \\
\hline & Yes & 39.1 (23.6) & & $59.8(22.0)$ & & $58.8(32.3)$ & & $42.5(28.3)$ & & 49.4 (19.5) & \\
\hline Antibodies & No & $44.9(20.6)$ & 0.521 & $60.4(21.5)$ & 0.359 & 59.1 (27.7) & 0.388 & $51.1(25.4)$ & 0.216 & $5 I .1(18.6)$ & 0.850 \\
\hline & Yes & $42.3(22.1)$ & & $63.9(16.5)$ & & $54.5(29.3)$ & & $44.9(29.7)$ & & $50.4(21.3)$ & \\
\hline Metastasis & No & $48.7(19.8)$ & $0.00 I^{*}$ & $63.2(20.8)$ & 0.101 & $63.2(26.4)$ & $0.00 I^{*}$ & $55.6(25.7)$ & $0.00 I^{*}$ & $55.9(18.0)$ & $0.001 *$ \\
\hline & Yes & $32.8(19.0)$ & & $57.3(17.7)$ & & $47.2(29.4)$ & & $34.0(22.5)$ & & $38.8(16.6)$ & \\
\hline Fever & No & 47.7 (19.5) & 0.004 & $66.5(16.4)$ & $0.001 *$ & $61.8(25.5)$ & 0.071 & $54.0(25.1)$ & $0.003 *$ & $54.5(19.4)$ & $0.002 *$ \\
\hline & Yes & $37.5(21.5)$ & & $52.9(22.8)$ & & $52.5(31.7)$ & & $40.6(27.2)$ & & $44.3(17.1)$ & \\
\hline Family support & No & $40.0(23.6)$ & 0.463 & $55.1(21.8)$ & 0.220 & $54.5(27.6)$ & 0.588 & 44.1 (23.7) & 0.470 & $48.2(16.1)$ & 0.613 \\
\hline & Yes & $44.3(20.5)$ & & $62.1(19.8)$ & & $58.8(28.4)$ & & $49.5(26.9)$ & & $51.0(19.5)$ & \\
\hline Chronic disease & No & $44.6(18.8)$ & 0.602 & $60.6(19.1)$ & 0.542 & $58.3(27.9)$ & 0.987 & $52.4(26.3)$ & 0.039 & $52.5(18.8)$ & 0.135 \\
\hline other than cancer & Yes & $42.6(24.1)$ & & $62.8(21.8)$ & & $58.4(29.0)$ & & $42.9(26.4)$ & & 47.5 (19.7) & \\
\hline Regular exercise & No & $41.9(21.3)$ & 0.085 & $59.8(21.0)$ & 0.157 & $56.7(29.3)$ & 0.297 & $45.7(27.2)$ & 0.025 & $47.2(19.3)$ & $0.001 *$ \\
\hline & Yes & $48.3(19.3)$ & & $64.9(17.4)$ & & $62.0(25.7)$ & & $56.3(24.0)$ & & $58.2(16.8)$ & \\
\hline
\end{tabular}

Note: *The variable is significant using the Bonferroni correction cutoff at $\alpha / n=0.05 / 19=0.003$, where $n$ is the number of tests.

Abbreviation: SD, standard deviation.

education levels. Elderly (age $\geq 60$ years) women reported poorer physical function than women of ages $<60$ years (mean difference $=-17.9, P=0.002$ ). Breast cancer patients with a university degree reported better physical function than those with a high school education or less (mean difference $=17.7$, $P=0.001$ ). These confirmed the findings from previous studies that QoL in breast cancer patients was negatively affected by patient age ${ }^{8,43}$ and positively affected by education levels. ${ }^{44,45}$

We noted that breast cancer patients who reported having metastasis and multiple tumors have pain, very poor physical functions, role limitations due to emotional problems, little vitality, low social functioning, and poor general health. Metastasis ${ }^{46,47}$ and multiple tumors ${ }^{48}$ have been linked with poor QoL in previous studies. According to our study, fever indicates considerable deterioration in the breast cancer patient's QoL. Fever tends to worsen several SF-36 domains: role limitations due to emotional problems, emotional wellbeing, pain, and general health. This could be due to the indirect effect of treatment on QoL, since research has indicated that fever may result from adverse treatment effects. ${ }^{49}$ 
Table 3 Multiple linear regression showing predictors of health-related quality of life in breast cancer patients

\begin{tabular}{|c|c|c|c|c|c|c|c|c|c|c|c|c|c|c|c|c|}
\hline \multirow[t]{2}{*}{ Characteristics } & \multicolumn{2}{|c|}{$\begin{array}{l}\text { Physical } \\
\text { functioning }\end{array}$} & \multicolumn{2}{|c|}{$\begin{array}{l}\text { Role } \\
\text { limitations } \\
\text { due to } \\
\text { physical } \\
\text { health }\end{array}$} & \multicolumn{2}{|c|}{$\begin{array}{l}\text { Role } \\
\text { limitations } \\
\text { due to } \\
\text { emotional } \\
\text { problems }\end{array}$} & \multicolumn{2}{|c|}{ Vitality } & \multicolumn{2}{|c|}{$\begin{array}{l}\text { Emotional } \\
\text { wellbeing }\end{array}$} & \multicolumn{2}{|c|}{$\begin{array}{l}\text { Social } \\
\text { functioning }\end{array}$} & \multicolumn{2}{|l|}{ Pain } & \multicolumn{2}{|c|}{$\begin{array}{l}\text { General } \\
\text { health }\end{array}$} \\
\hline & B & $P$-value & B & $P$-value & B & $P$-value & B & $P$-value & B & $P$-value & B & $P$-value & B & $P$-value & B & $P$-value \\
\hline Constant & 66.1 & 0.001 & -15.1 & 0.048 & 50.0 & 0.001 & 55.7 & 0.001 & 70.3 & 0.001 & 103.6 & 0.001 & 60.6 & 0.001 & 58.1 & 0.001 \\
\hline Age & & & & & & & & & & & -0.4 & 0.035 & & & & \\
\hline University & 13.4 & 0.013 & & & & & & & & & -16.5 & 0.002 & & & & \\
\hline Employed & & & & & -20.4 & 0.018 & -9.4 & 0.018 & -10.1 & 0.009 & & & & & & \\
\hline \multicolumn{17}{|l|}{ Married } \\
\hline \multicolumn{17}{|l|}{ Obese } \\
\hline \multicolumn{17}{|l|}{ of cancer } \\
\hline \multicolumn{17}{|l|}{ cancer diagnosis } \\
\hline \multicolumn{17}{|l|}{ Cancer stage } \\
\hline Multiple tumors & & & & & -31.6 & 0.001 & & & & & -20.6 & 0.001 & & & & \\
\hline Cancer surgery & -10.8 & 0.047 & & & & & & & & & & & & & & \\
\hline \multicolumn{17}{|l|}{ Chemotherapy } \\
\hline \multicolumn{17}{|l|}{ Radiation therapy } \\
\hline Immunotherapy & & & & & & & -7.2 & 0.046 & & & & & -10.8 & 0.020 & & \\
\hline \multicolumn{17}{|l|}{ Antibodies } \\
\hline Metastasis & -22.9 & 0.001 & & & & & -15.0 & 0.001 & & & & & -19.4 & 0.001 & -16.9 & 0.001 \\
\hline Fever & & & & & & & & & -9.1 & 0.011 & -11.0 & 0.026 & & & & \\
\hline \multicolumn{17}{|l|}{ Family support } \\
\hline \multicolumn{17}{|l|}{ Chronic disease } \\
\hline \multicolumn{17}{|l|}{ other than } \\
\hline \multicolumn{17}{|l|}{ cancer } \\
\hline Regular exercise & & & & & & & & & & & & & & & 8.2 & 0.017 \\
\hline \multicolumn{17}{|c|}{ Model summary } \\
\hline$F$-value & 10.1 & 0.001 & 4.0 & 0.048 & 9.9 & 0.001 & 8.9 & 0.001 & 7.0 & 0.001 & 7.0 & 0.001 & 13.1 & 0.001 & 12.1 & 0.001 \\
\hline$R^{2}$ & 0.22 & & 0.04 & & 0.16 & & 0.20 & & 0.17 & & 0.26 & & 0.20 & & 0.26 & \\
\hline$R$ & 0.47 & & 0.19 & & 0.40 & & 0.45 & & $0.4 \mathrm{I}$ & & 0.51 & & 0.45 & & 0.51 & \\
\hline
\end{tabular}

Notes: B represents the partial regression coefficient. F-value represents the test value of overall significance of the linear regression model; $R^{2}$ represents the proportion of variance explained; $R$ represents multiple correlation coefficient.

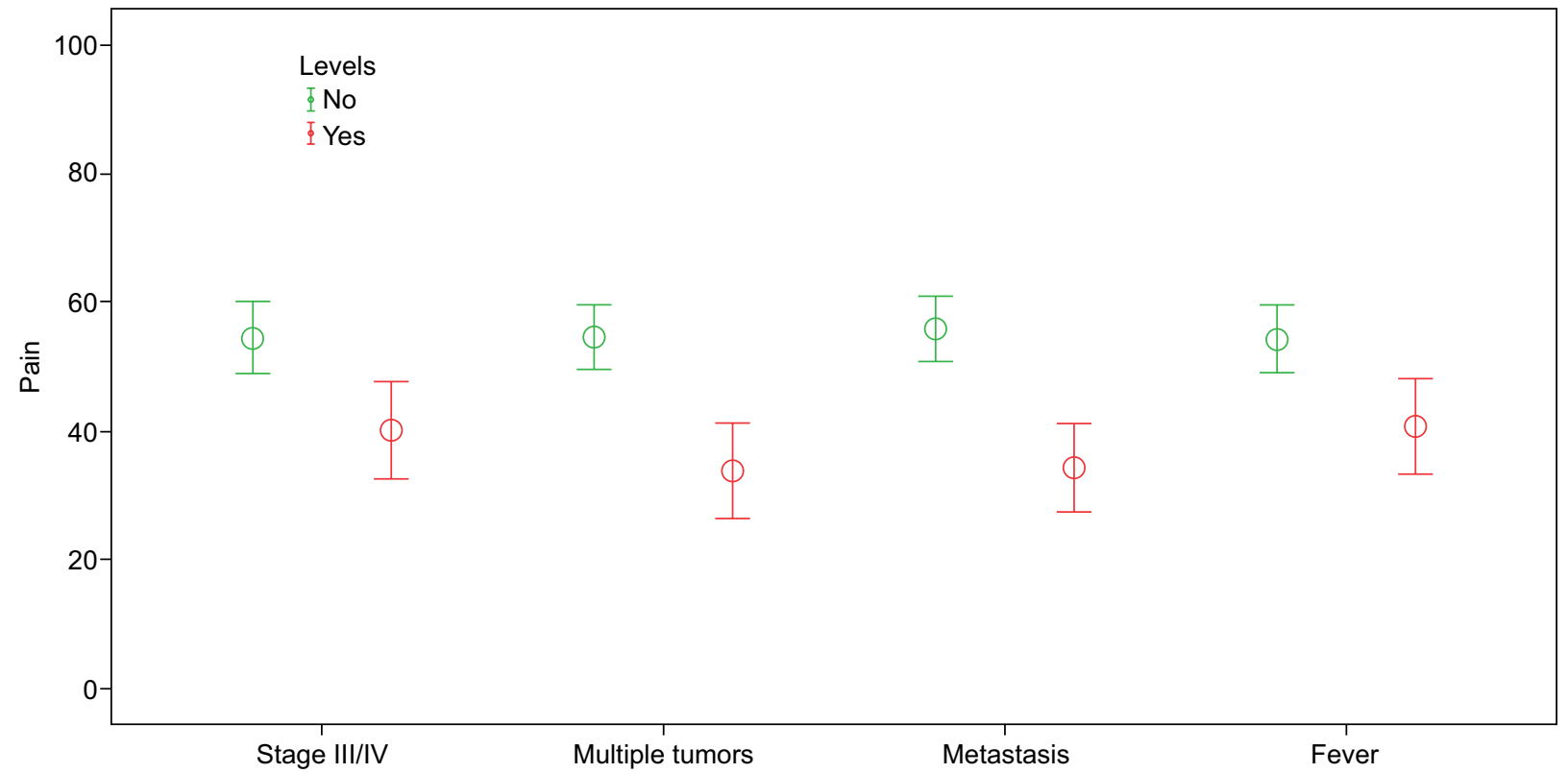

Figure I Error bar charts, impact of cancer complications on bodily pain ratings.

Note: The higher the score, the lesser the pain. 


\section{Limitations}

We noted several limitations in conducting this research: findings must be interpreted with caution as the cross-sectional study assesses associations and may not allow causality. There is a potential for selection bias, in that patients who are attending our Oncology Outpatient Clinic may more often be likely to participate in the study, given the perceived severity of their cancer. The Generic SF-36 questionnaire was used to measure QoL and not as a breast cancer-specific measure. In our population, SF-36 appears to be a reliable assessment of QoL, and this research has clearly identified several factors that appear to affect QoL in breast cancer patients. This study is of interest to QoL researchers, cancer researchers, providers caring for cancer patients, and cancer patients. This study could be of value to the kingdom's health system in promoting the QoL of Saudi women with breast cancer by introducing educational programs or by endorsing/sponsoring public health policies.

\section{Conclusion}

In Saudi breast cancer patients, regular exercise was a significant positive predictor of increased general health. In several SF-36 domains, Saudi breast cancer patients with multiple tumors, metastasis, or fever tend to experience significantly poor QoL. For such breast cancer patients, the effectiveness of interventional programs, such as regular exercise, should be assessed to maintain and improve QoL. Thus, it is important to provide routine assessment of QoL in breast cancer patients.

\section{Acknowledgments}

This study was supported by King Abdullah International Medical Research Center (KAIMRC) as part of 8th Research Summer School (2016). The authors would like to thank KAIMRC Research Summer School Team for giving us the opportunity to conduct this research.

\section{Disclosure}

The authors report no conflicts of interest in this work.

\section{References}

1. Daudt A, Alberg AJ, Helzlsouer KJ. Epidemiology, prevention, and early detection of breast cancer. Current Opin Oncol. 1996;8(6):455-461.

2. Tarone RE. Breast cancer trends among young women in the United States. Epidemiology. 2006;17(5):588-590.

3. Saudi Cancer Registry 1996. Saudi Cancer Registry, Saudi Oncology Society. Available from: http://shc.gov.sa/En/HealthRecords/CancerRegistry/Pages/CancerRegistryRecords.aspx. Accessed September 12, 2016.

4. Saudi Cancer Registry 2012. Saudi Cancer Registry, Saudi Oncology Society. Available from: http://shc.gov.sa/En/HealthRecords/CancerRegistry/Pages/CancerRegistryRecords.aspx. Accessed September 12, 2016.
5. Cella DF, Cherin EA. Quality of life during and after cancer treatment. Comp Ther. 1988;14(5):69-75.

6. Shimozuma K, Ganz PA, Petersen L, Hirji K. Quality of life in the first year after breast cancer surgery: rehabilitation needs and patterns of recovery. Breast Cancer Res Treat. 1999;56(1):45-57.

7. Lindley C, Vasa S, Sawyer WT, Winer EP. Quality of life and preferences for treatment following systemic adjuvant therapy for early-stage breast cancer. J Clin Oncol. 1998;16(4):1380-1387.

8. King MT, Kenny P, Shiell A, Hall J, Boyages J. Quality of life three months and one year after first treatment for early stage breast cancer: influence of treatment and patient characteristics. Qual Life Res. 2000; 9(7):789-800.

9. Janni W, Rjosk D, Dimpfl TH, et al. Quality of life influenced by primary surgical treatment for stage I-III breast cancer-long-term follow-up of a matched-pair analysis. Ann Surg Oncol. 2001;8(6):542-548.

10. Avis NE, Crawford S, Manuel J. Quality of life among younger women with breast cancer. J Clin Oncol. 2005;23(15):3322-3330.

11. Reich M, Lesur A, Perdrizet-Chevallier C. Depression, quality of life and breast cancer: a review of the literature. Breast Cancer Res Treat. 2008;110(1):9-17.

12. Burgess C, Cornelius V, Love S, Graham J, Richards M, Ramirez A. Depression and anxiety in women with early breast cancer: five year observational cohort study. BMJ. 2005;330(7493):702.

13. Cooper CL, Faragher EB. Psychosocial stress and breast cancer: the interrelationship between stress events, coping strategies and personality. Psychol Med. 1993;23(03):653-662.

14. Lueboonthavatchai P. Prevalence and psychosocial factors of anxiety and depression in breast cancer patients. J Med Assoc Thai. 2007;90(10):2164-2174.

15. Cohen M, Mabjish AA, Zidan J. Comparison of Arab breast cancer survivors and healthy controls for spousal relationship, body image, and emotional distress. Qual Life Res. 2011;20(2):191-198.

16. Goldblatt H, Cohen M, Azaiza F. Expression of emotions related to the experience of cancer in younger and older Arab breast cancer survivors. Ethn Health. 2016;21(6):564-577.

17. El Sharkawi FM, Sakr MF, Atta HY, Ghanem HM. Effect of different modalities of treatment on the quality of life of breast cancer patients in Egypt. La Revue de Santé de la Méditerranée orientale. 1997;3(1): 68-81.

18. Mostafa ESM, Sadek RR, El-Sherif MA, Saber RA, Mosalem FA. Assessment of quality of life for female breast cancer patients attending el-minia oncology center from June to December 2009. El-Minia Med Bul. 2010;21(1):161-170. Lithuanian.

19. Denewer A, Farouk O, Kotb S, Setit A, Abd Elkhalek S, Shetiwy M. Quality of life among Egyptian women with breast cancer after sparing mastectomy and immediate autologous breast reconstruction: a comparative study. Breast Cancer Res Treat. 2012;133(2):537-544.

20. Awad MA, Denic S, El Taji H. Validation of the European organization for research and treatment of cancer quality of life questionnaires for Arabic-speaking populations. Ann N Y Acad Sci. 2008;1138: $146-154$.

21. Alawadi SA, Ohaeri JU. Health - related quality of life of Kuwaiti women with breast cancer: a comparative study using the EORTC quality of life questionnaire. BMC Cancer. 2009;9:222.

22. Masmoudi A, Frikha M, Daoud J. Feasibility of quality of life assessment in routine clinical oncology practice: a Tunisian study. East Mediterr Health J. 2009;15(2):362-368.

23. Ba-Khubaira S, Al-Kahiry W. Age related quality of life among selected breast cancer patients in Aden, Yemen. Pan Arab J Oncol. 2012;5(1) 14-17.

24. Abu-Saad Huijer H, Abboud S. Health-related quality of life among breast cancer patients in Lebanon. Eur J Oncol Nurs. 2012;16(5): 491-497.

25. Jassim GH, Whitford DL. Quality of life of Bahraini women with breast cancer: a cross sectional study. BMC Cancer. 2013;13:212.

26. Jassim GH, Whitford DL. Understanding the experiences and quality of life issues of Bahraini women with breast cancer. Soc Sci Med. 2014;107:189-195. 
27. El Fakir S, Abda N, Bendahhou K, et al. The European organization for research and treatment of cancer quality of life questionnaire-BR 23 breast cancer-specific quality of life questionnaire: psychometric properties in a Moroccan sample of breast cancer patients. BMC Res Notes. 2014;7:53.

28. Abu-Helalah M, Al-Hanaqta M, Alshraideh H, Abdulbaqi N, Hijazeen J. Quality of life and psychological well-being of breast cancer survivors in Jordan. Asian Pac J Cancer Prev. 2014;15(14):5927-5936.

29. Almutairi KM, Mansour EA, Vinluan JM. A cross-sectional assessment of quality of life of breast cancer patients in Saudi Arabia. Public Health. 2016;136:117-125

30. Habib F, Salman S, Safwat M, Shalaby S. Awareness and knowledge of breast cancer among university students in Al Madina Al Munawara Region. Middle East J Cancer. 2010;1(4):159-166.

31. Radi SM. Breast cancer awareness among Saudi females in Jeddah. Asian Pac J Cancer Prev. 2013;14(7):4307-4312.

32. Sait WA, Al-Amoudi SM, Tawtai DA, Abduljabbar HS. The knowledge of breast cancer among young Saudi females. Saudi Med J. 2010;31(11):1242-1244.

33. Alam AA. Knowledge of breast cancer and its risk and protective factors among women in Riyadh. Ann Saud Med. 2006;26(4):272-277.

34. Amin TT, Al Mulhim AR, Al Meqihwi A. Breast cancer knowledge, risk factors and screening among adult Saudi women in a primary health care setting. Asian Pac J Cancer Prev. 2009;10(1):133-138.

35. Milaat WA. Knowledge of secondary-school female students on breast cancer and breast self-examination in Jeddah, Saudi Arabia. East Mediterr Health J. 2000;6(2-3):338-344.

36. Jahan S, Al-Saigul AM, Abdelgadir MH. Breast cancer. Knowledge, attitudes and practices of breast self examination among women in Qassim region of Saudi Arabia. Saudi Med J. 2006;27(11):1737-1741.

37. Dandash KF, Al-Mohaimeed A. Knowledge, attitudes, and practices surrounding breast cancer and screening in female teachers of Buraidah, Saudi Arabia. Int J Health Sci (Qassim). 1(1):61-71.

38. Taouk M, Lovibond PF, Laube R. Psychometric properties of an Arabic version of the Depression Anxiety Stress Scales (DASS21). Report for New South Wales Transcultural Mental Health Centre, Cumberland Hospital, Sydney; 2001.
39. Coons SJ, Alabdulmohsin SA, Draugalis JR, Hays RD. Reliability of an Arabic version of the RAND 36-item health survey and its equivalence to the US-English version. Med Care. 1998;36(3):428-432.

40. Ahmed AE, Alaskar AS, Al-Suliman AM, et al. Health-related quality of life in patients with sickle cell disease in Saudi Arabia. Health Qual Life Outcomes. 2015;13(1):183.

41. Lu Q, You J, Kavanagh A, et al. Differences in quality of life between American and Chinese breast cancer survivors. Support Care Cancer. 2016;24(9):3775-3782.

42. Høyer M, Johansson B, Nordin K, et al. Health-related quality of life among women with breast cancer-a population-based study. Acta Oncol. 2011;50(7):1015-1026.

43. Ganz PA, Lee JJ, Sim MS, Polinsky ML, Schag CA. Exploring the influence of multiple variables on the relationship of age to quality of life in women with breast cancer. J Clin Epidemiol. 1992;45(5): 473-485.

44. Ashing Giwa K, Ganz PA, Petersen L. Quality of life of African American and white long term breast carcinoma survivors. Cancer. 1999;85(2):418-426.

45. Peuckmann V, Ekholm O, Rasmussen NK, et al. Health-related quality of life in long-term breast cancer survivors: nationwide survey in Denmark. Breast Cancer Res Treat. 2007;104(1):39-46.

46. Diel IJ, Body JJ, Lichinitser MR, et al; MF 4265 Study Group. Improved quality of life after long-term treatment with the bisphosphonate ibandronate in patients with metastatic bone disease due to breast cancer. Eur J Cancer. 2004;40(11):1704-1712.

47. Wyatt G, Sikorskii A, Tamkus D, You M. Quality of life among advanced breast cancer patients with and without distant metastasis. Eur J Cancer Care (Engl). 2013;22(2):272-280.

48. Tang Z, Wang J, Zhang H, et al. Associations between diabetes and quality of life among breast cancer survivors. PLoS One. 2016; 11(6):e0157791

49. Lipton A, Theriault RL, Hortobagyi GN, et al. Pamidronate prevents skeletal complications and is effective palliative treatment in women with breast carcinoma and osteolytic bone metastases. Cancer. 2000; 88(5):1082-1090.
Breast Cancer - Targets and Therapy

\section{Publish your work in this journal}

Breast Cancer - Targets and Therapy is an international, peerreviewed open access journal focusing on breast cancer research, identification of therapeutic targets and the optimal use of preventative and integrated treatment interventions to achieve improved outcomes, enhanced survival and quality of life for the cancer patient.

\section{Dovepress}

The manuscript management system is completely online and includes a very quick and fair peer-review system, which is all easy to use. Visit http://www.dovepress.com/testimonials.php to read real quotes from published authors. 